\title{
La santé des adolescentes vulnérables : un investissement stratégique doublement rentable
}

Jennifer Catino

Population Council

Follow this and additional works at: https://knowledgecommons.popcouncil.org/departments_sbsr-pgy

Part of the Family, Life Course, and Society Commons, Gender and Sexuality Commons, International Public Health Commons, and the Women's Health Commons How does access to this work benefit you? Let us know!

\section{Recommended Citation}

Catino, Jennifer. 2012. "La santé des adolescentes vulnérables : un investissement stratégique doublement rentable," GIRLS FIRST ! Perspectives de programmation centrée sur les filles. New York: Population Council. 
UNE PUBLICATION GIRLS FIRST!

LA SANTÉ DES ADOLESCENTES VULNÉRABLES : UN INVESTISSEMENT STRATÉGIQUE DOUBLEMENT RENTABLE

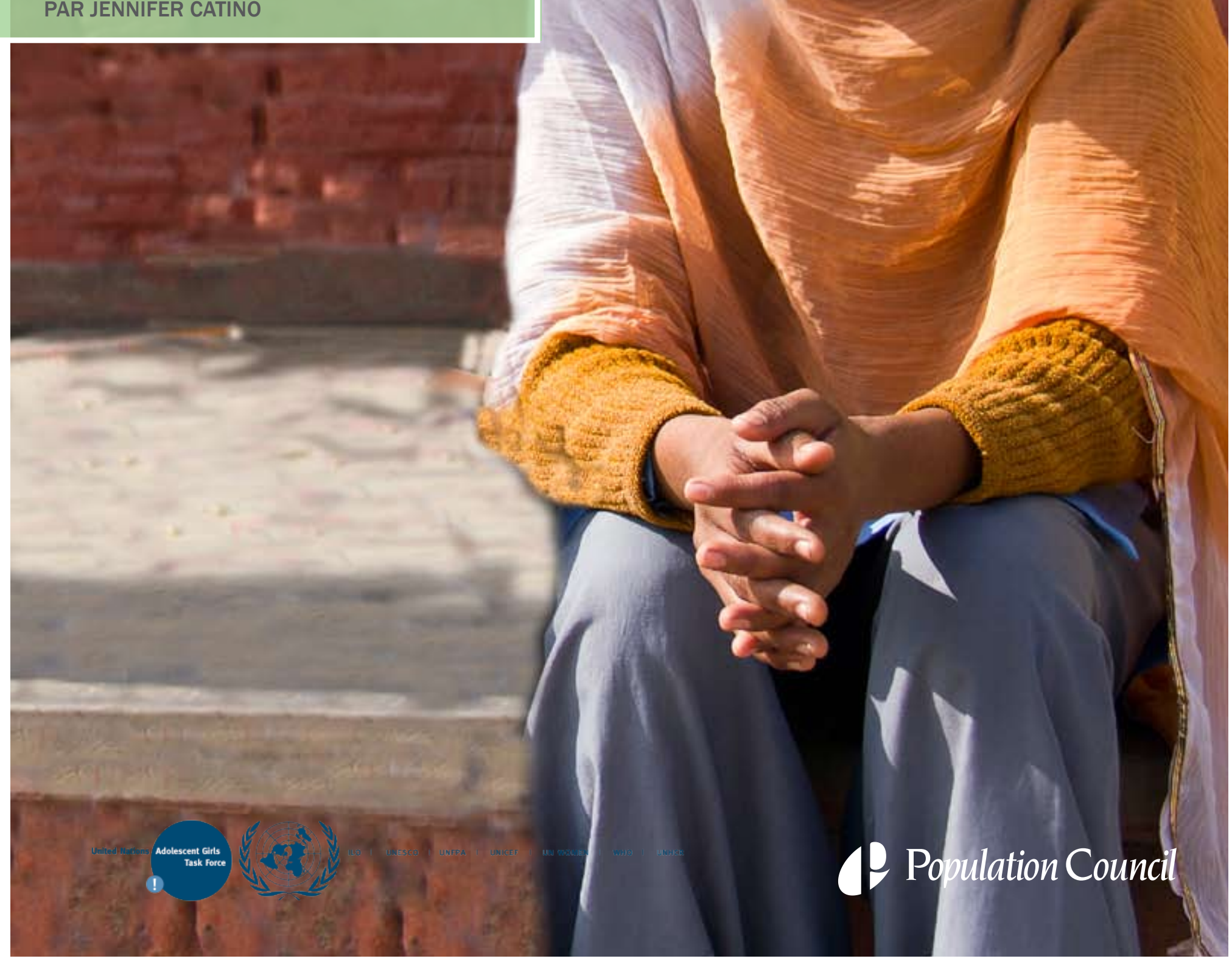




\section{GIRLS FIRST!}

\section{PERSPECTIVES DE PROGRAMMATION CENTRÉE SUR LES FILLES}

La recherche et les programmes consacrés aux adolescentes se concentrent traditionnellement sur la sexualité, la santé génésique et le comportement, négligeant la problématique socioéconomique plus large qui sous-tend leurs droits humains, leur développement général, leur santé et leur bienêtre. De plus, les efforts d'amélioration de la vie des filles mettent souvent en vedette ceux et celles qui dominent ou influencent leur vie (les parents, la belle-famille, les garçons, les hommes, les agresseurs), sans prêter aux intéressées elles-mêmes l'attention qu'elles méritent en soi.

GIRLS FIRST! Perspectives de programmation centrée sur les filles est une série de cinq comptes rendus thématiques, rédigés par les experts et expertes du Population Council. Ces dossiers présentent un instantané de la base de connaissances telle qu'elle se présente, à un moment donné, dans ce domaine en évolution permanente. Ils abordent les cinq priorités stratégiques définies dans la Déclaration conjointe des Nations Unies, "Intensifier l'action menée pour promouvoir les droits des adolescentes " (mars 2010), symbole de l'engagement collectif de sept organismes de l'ONU au soutien des gouvernements et de leurs partenaires pour la promotion de politiques et programmes essentiels au profit des adolescentes les plus difficiles à atteindre. Ces comptes rendus

1. explorent l'évolution prochaine de l'éducation des filles ;

2. définissent les grandes lignes d'approches innovantes en vue de l'amélioration de la santé des filles;

3. recadrent l'approche de la violence à l'encontre des filles ;

4. décrivent les meilleurs moyens de cultiver les leaders féminines; et

5. expliquent de nouveaux modes inédits de collecte et d'exploitation des données relatives aux adolescentes.

Ils avancent des arguments novateurs en faveur de l'investissement dans les filles et mettent en valeur les pratiques prometteuses. Ils expriment un point de vue factuel progressiste quant aux allocations de ressources aptes à assurer le plus rapidement et le plus efficacement l'amélioration de la vie des filles.

Rédigés par des experts et expertes du Population Council, une organisation pionnière d'une recherche et programmation de pointe à l'intention des adolescentes vulnérables et marginalisées, ces rapports ont bénéficié, d'abord et surtout, de l'appui et du leadership de l'Équipe spéciale des Nations Unies pour les adolescentes. L'initiative a également reçu l'appui moral et financier de la Nike Foundation, de la Fondation des Nations Unies et de la David and Lucile Packard Foundation. Ces comptes rendus viennent s'ajouter à la guidance programmatique et aux " boîtes à outils " aujourd'hui disponibles ; ils offrent une ressource de référence essentielle à toute initiative d'élaboration de politiques et programmes efficaces et durables au profit des filles. Notre espoir est qu'ils inspireront de nouvelles approches utiles aux efforts de réalisation des droits des adolescentes marginalisées du monde.

Juillet 2012

Population Council et Équipe spéciale des Nations Unies pour les adolescentes

Avis

Ces comptes rendus sur la programmation pour adolescentes dans les domaines de l'éducation, de la santé, de la réduction de la violence, du leadership des filles et des données ont été préparés par le Population Council pour l'Équipe spéciale des Nations Unies pour les adolescentes, avec l'appui de la Nike Foundation, de la Fondation des Nations Unies et de la David and Lucile Packard Foundation.

Les opinions exprimées sont strictement celles des auteurs et ne reflètent pas nécessairement celles des partenaires donateurs.

(c) 2012 The Population Council, Inc. 


\title{
LA SANTÉ DES ADOLESCENTES VULNERABLES : UN INVESTISSEMENT STRATEGIQUE DOUBLEMENT RENTABLE
}

\author{
PAR JENNIFER CATINO
}

INTRODUCTION ET PRÉMISSES …………………......... 2 ÉTAT ACTUEL DES CHOSES .......................................... 2

Deux fondements de la santé ........................................... 2

Progrès de la santé infantile ces 30 dernières années : beaucoup de filles en ont bénéficié, mais beaucoup aussi ont été laissées pour compte.

Chez les adolescentes, la maladie et la perte de l'intégrité physique sont d'origine sociale et s'amplifient à la puberté. 4

Certains risques de santé et sociaux associés affectent exclusivement ou principalement les adolescentes.

Les comportements de santé établis à l'adolescence peuvent affecter toute la vie, non seulement des filles et des femmes mais aussi des générations futures

Les systèmes de santé ne répondent pas aux besoins des adolescentes vulnérables - en tant que consommatrices ou que prestataires de soins et services

POLITIQUES ET PRATIQUES PRÉCONISÉES. 8

Mener une recherche ciblée sur les problèmes de santé particuliers ou partagés des adolescentes vulnérables

Cartographier l'état de santé des filles et leurs expériences sociales étroitement liées pour comprendre les vulnérabilités spécifiques à l'âge, au genre et au contexte des sous-groupes de filles et jeunes femmes........ 8

Mener une recherche participative et tester les stratégies pour mieux comprendre et soutenir le double rôle des filles et des jeunes femmes en tant que consommatrices et prestataires de soins de santé

Atténuer les risques de santé que font courir aux adolescentes le manque de sécurité et la fréquence des menaces ou de l'expérience de la violence

Développer la capacité de réflexion critique des adolescentes concernant leur santé et leur vie. 9

Fournir aux médias des scénarios plus spécifiques concernant les risques posés à la santé des adolescentes et promouvoir une image positive et réaliste des filles........ 9

Identifier et tester les possibilités de recours aux technologies de plus en plus accessibles pour améliorer la santé et la sécurité des filles. . .9

Instaurer des programmes qui ciblent et soutiennent la transition critique de la puberté
Soutenir les mères primipares les plus jeunes, qui portent le fardeau d'une part disproportionnée et grandissante de la mauvaise santé maternelle. 10

En partant des filles, chercher à mieux comprendre et mesurer l'engagement positif des garçons et des jeunes hommes au soutien de la santé et du bien-être des filles vulnérables

Mobiliser les filles et les jeunes femmes pour renforcer le secteur de la santé et élargir leurs horizons de subsistance

Informer les filles et les jeunes femmes pour qu'elles comprennent leurs droits et leurs responsabilités en leur qualité de consommatrices comme de prestataires de soins de santé

\section{RESSOURCES : PROGRAMMES « LABORATOIRES D'APPRENTISSAGE ", PUBLICATIONS ET OUTILS ....12}

Abriendo Oportunidades: Travailler avec les filles du Guatemala rural pour découvrir leur potentiel de santé et réduire la violence

Rwanda 12+ : Concentrer une attention systématique sur le potentiel et les besoins de la fille de 12 ans

Berhane Hewan : Créer un espace de soutien des filles non mariées et mariées et des plus jeunes mères primipares

Puntos de Encuentro: Au carrefour de la violence et de la santé précaire.

Un seul programme : Guide pour une approche pédagogique unifiée de la sexualité, du genre, du VIH et des droits humains.

Investing When It Counts: Generating the Evidence Base for Policies and Programs for Very Young Adolescents

Guide pratique de l'OMS pour les soins aux adolescents : Un outil de référence destiné aux agents de santé de premier niveau

Conception d'un programme centré sur les filles :

Boîte à outils pour l'élaboration, le renforcement et l'expansion de programmes destinés aux adolescentes..... 14

Donner aux jeunes femmes le pouvoir d'initier le changement 14

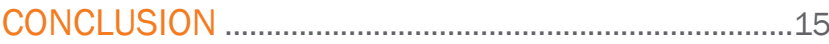

NOTES AND RÉFÉRENCES .........................................15 


\section{INTRODUCTION ET} PRÉMIISSES

Le monde compte aujourd'hui 1,2 milliard d'adolescents âgés de 10 à 19 ans. Près de 90\% d'entre eux vivent dans les pays en développement et environ 600 millions sont de sexe féminin ${ }^{1}$. L'adolescence représente normalement une période de santé florissante bien que sujette à de nombreux changements socio-développementaux dont les implications affectent, tout particulièrement, la santé des filles. La plupart des centaines de millions de filles et jeunes femmes du monde en développement vivent dans des conditions et des circonstances qui les rendent vulnérables aux risques de santé et sociaux. Ces adolescentes sont souvent pauvres, déscolarisées, mariées, migrantes, membres de minorités ethniques ou sujettes à des conditions de travail dangereuses.

Le lot des filles varie radicalement suivant le contexte. Dans beaucoup des communautés les plus démunies du monde, elles restent la proie de maladies transmissibles, de la dénutrition et de pratiques traditionnelles néfastes. Ailleurs, elles présentent des taux d'obésité, de tabagisme et de dépression en hausse. Transmissibles ou non, les maladies qui les affectent nécessitent une approche multisectorielle globale, qui aide à comprendre les besoins de santé et sociaux interdépendants des filles et à y répondre.

Dans cette optique, les approches qui apportent aux filles une connaissance, des compétences et des ressources intégrales - au niveau personnel aussi bien que de l'environnement dans lequel elles évoluent - joueront un rôle important dans leur accès à la prise en charge de leur corps et de leur vie. Le secteur de la santé doit s'améliorer et inclure davantage les filles pour maximiser son impact. Les autres secteurs doivent aussi se mobiliser au soutien des filles et les aider à opérer les choix et à rechercher les occasions propices à leur santé et à la pleine réalisation de leur potentiel.
L'accent est mis ici sur les risques de santé et les besoins des adolescentes vulnérables, ainsi que sur les vastes horizons qui s'ouvrent à elles lorsque sont consentis les investissements stratégiques favorables à leur santé, leur bien-être et leurs capacités de résolution de leurs problèmes.

Les données éventuellement disponibles doivent être intégrées et mises à profit. La documentation factuelle relative aux besoins de santé spécifiques et aux solutions requises pour les sous-groupes vulnérables d'adolescentes est cependant maigre (les données généralement collectées sur la santé des adolescents sont limitées et rarement ventilées en fonction du sexe, de l'âge ou d'autres facteurs) ${ }^{2,3}$.

L'objectif de ce compte rendu est double en ce qu'il entend examiner plus avant les défis de santé posés aux filles et détailler la promesse de l'investissement dans ces mêmes filles. L'accent est mis sur les idées et initiatives innovantes aptes à guider le secteur vers une meilleure réponse et maximisation du potentiel des filles les plus pauvres des communautés les plus démunies. La recherche indique de plus en plus que le progrès sanitaire et socioéconomique sera plus rapide et plus rentable si l'on se concentre d'abord sur les filles les plus pauvres des milieux les plus difficiles d'accès. Donner la priorité à l'équité dans la réalité mondiale de l'incertitude économique n'est pas seulement une approche juste : c'est une approche qui marche $e^{4}$.

\section{ÉTAT ACTUEL DES CHOSES}

\section{Deux fondements de la santé}

L'adolescence est la période durant laquelle s'établissent deux grands fondements de la santé des filles:

- Leur propre santé personnelle en ce qui concerne leur activité sexuelle et leur sentiment de contrôle de leur corps, de leur alimentation et de leur activité physique. Les tendances comportementales profondes qui affec- tent la santé s'établissent souvent à l'adolescence. Elles sont souvent dictées par le milieu socioéconomique. L'éducation, la santé et le bien-être des adolescentes influencent du reste ceux des enfants qu'elles auront.

- Leur aptitude à voir en leur propre personne les agents du changement en ce qui concerne leur propre santé et celle d'autrui.

L'adolescence est une période où les compétences, les connaissances et l'éducation des filles doivent les préparer à gagner sainement leur vie. Apprendre aux filles à reconnaître et confronter les problèmes de santé présents dans leurs communautés jette les bases de leur participation et les mobilise à devenir partie intégrante de la solution à leurs propres besoins de santé et à ceux de la communauté.

Par exemple, les adolescentes brevetées du programme Abriendo Oportunidades au Guatemala rural (jeunes femmes de 16 ans et plus) sont prêtes à apprendre et à appliquer de plus profondes compétences tout en assumant de nouveaux rôles de leadership au sein de leurs communautés. Parce que ces jeunes femmes partagent la culture et la langue indigène et parce qu'elles sont devenues les ressources connues et fiables des membres de la communauté, elles sont stratégiquement positionnées pour offrir une information utile sur les soins de santé primaires, les produits élémentaires et l'orientation vers les services de santé. En coopération avec les systèmes de santé communautaire, le programme a entrepris un essai pilote de formation et soutien de ses jeunes brevetées pour qu'elles assument un rôle de promotion de la santé au sein de leurs familles et communautés, comblant ainsi un manque profond de ressources humaines dans les milieux ruraux tout en dotant les jeunes femmes de compétences professionnelles, d'une expérience utile et de débouchés de subsistance.

Le Tableau 1 présente une liste d'exemples concrets de formation, soutien et rémunération des adolescentes à l'apport de solutions de santé. 


\section{TABLEAU 1 SOLUTIONS DE SANTÉ COMMUNAUTAIRE DÉLIVRÉES PAR LES ADOLESCENTES}

\section{PROBLÈMES DE SANTÉ COMMUNAUTAIRE}

Maladie diarrhéique

Affection respiratoire

Malnutrition

Paludisme

Grossesse non désirée

Mortalité maternelle

Violence de genre

Mortalité infantile

VIH/IST

Obésité

Dépression/santé mentale

Abus de substances toxiques

\section{SOLUTION DÉLIVRÉE PAR LES ADOLESCENTES}

Promotion de l'hygiène, apport d'eau propre, reconnaissance des symptômes, distribution de SRO, orientation opportune vers les services de santé appropriés.

Promotion d'un environnement propre, reconnaissance des symptômes, orientation opportune vers les services appropriés.

Promotion d'une alimentation équilibrée et préparation d'aliments sains, reconnaissance des symptômes, distributions de compléments alimentaires et de vitamines, direction de potagers familiaux et communautaires.

Promotion d'un environnement sain, distribution de moustiquaires, reconnaissance des symptômes, orientation opportune vers les services appropriés.

Apport d'information préventive complète, distribution de préservatifs et de certains contraceptifs, orientation opportune vers les services appropriés, animation de clubs de renforcement des compétences des filles célibataires et mariées.

Reconnaissance des indices de danger, orientation opportune vers les services appropriés, animation de clubs de renforcement des compétences des filles célibataires et mariées.

Apport d'information préventive, reconnaissance des indices et des zones de danger, orientation opportune vers les services appropriés, animation de clubs destinés à renforcer les capacités des filles à se protéger, élaboration de plans de sécurité.

Reconnaissance des indices de danger, orientation opportune vers les services appropriés.

Apport d'information préventive, distribution de préservatifs, orientation opportune vers les services de dépistage, conseil, soins et traitement.

Apport d'information préventive, promotion d'une alimentation équilibrée et de l'activité physique, orientation opportune vers les services appropriés, animation de clubs destinés à renforcer les compétences des filles.

Reconnaissance des indices de danger, orientation opportune vers les services appropriés, animation de clubs destinés à renforcer les compétences des filles, soutien social et entraide.

Reconnaissance des indices de danger, orientation opportune vers les services appropriés, animation de clubs destinés à renforcer les compétences des filles, soutien social et entraide.
Progrès de la santé infantile ces 30 dernières années : beaucoup de filles en ont bénéficié, mais beaucoup aussi ont été laissées pour compte.

La survie infantile a enregistré une hausse spectaculaire ces 30 dernières années. Depuis 1950, le taux de mortalité infantile a diminué d'environ $60 \%$ du fait de l'amélioration des conditions socio-sanitaires 5 . Combinée à la fécondité élevée qui persiste dans de nombreuses régions du monde, cette tendance veut dire que de plus en plus d'enfants, filles et garçons, accèdent désormais à l'adolescence ${ }^{6}$.

À l'âge de 10 ans, la plupart des filles du monde sont toujours scolarisées. Ces 10 dernières années ont été le témoin d'un progrès remarquable en termes de scolarisation primaire des filles, beaucoup de régions atteignant aujourd'hui la parité de genre7. La scolarisation des filles à l'adolescence est liée à une première expérience sexuelle différée, au mariage et à la maternité moins précoces, à de moindres taux de $\mathrm{VIH} /$ sida et autres morbidités sexuelles et génésiques et à un plus haut niveau d'égalité des sexes ${ }^{8}$. Les créneaux d'éducation des filles, de même que leur sécurité et leur santé dans le contexte scolaire ou hors école, tendent 
toutefois à faiblir lorsqu'elles atteignent l'âge de l'enseignement secondaire ${ }^{9}$, à l'approche critique de la puberté.

Chez les adolescentes, la maladie et la perte de l'intégrité physique sont d'origine sociale et s'amplifient à la puberté.

L'adolescence se caractérise normalement par de faibles niveaux de maladie et de mort. Jusqu'à la puberté, dans la plupart des régions du monde, les filles comme les garçons profitent de meilleures perspectives d'instruction, de santé et d'épanouissement personnel ${ }^{10}$. Si les filles ne sont pas en bonne santé à l'âge de 12 ans - et beaucoup ne le sont pas, surtout dans les communautés les plus pauvres du monde -, les raisons en sont principalement sociales et liées à la discrimination de genre.

Vers l'âge de 12 ans, les chemins des filles pauvres et des garçons commencent souvent à se séparer : les possibilités d'avancement des garçons continuent à s'étendre, tandis que celles des filles se rétrécissent souvent. La problématique de genre qui affecte spécifiquement les filles vers le moment de la puberté est illustrée au tableau présenté ci-après. Dès l'instant où elles sont réglées, les adolescentes accèdent souvent au rang de femmes adultes et sont soumises à une discrimination de genre accrue pouvant mener à leur déscolarisation, à l'accroissement de leur tâches ménagères, à la perte de leur réseau de pairs et à un isolement social accru, à la pression d'un mariage précoce relevant souvent d'une stratégie de survie économique et à une violence accrue.

"Ce qui arrive à la santé de la fille vers l'âge de 12 ans détermine son avenir - et celui de sa famille, de sa communauté et de son pays ${ }^{11}$."

Beaucoup des conséquences du changement physique, affectif et social rapide auquel se trouvent confrontées les adolescentes vulnérables sont ancrées dans l'inégalité de genre et la pauvreté. Ces conséquences contribuent à faire peser sur leur santé la menace de risques particuliers étroitement liés et souvent graves - qui affectent non seulement le bien-être et le développement des filles et des jeunes femmes en soi, mais aussi ceux de leurs enfants, des communautés et des nations.

Certains risques de santé et sociaux associés affectent exclusivement ou principalement les adolescentes.

La gestion de la menstruation peut représenter un problème écrasant pour les adolescentes pauvres. Dans les milieux à faibles ressources, les filles ne disposent pas des fournitures ou des installations qui leur permettraient de fréquenter l'école, de travailler ou de se déplacer librement et confortablement pendant leurs règles. La gêne, physique et autre, et la menace de la grossesse une fois la fille réglée se combinent souvent pour exclure les filles de la sphère publique et les réduire à la claustration des tâches ménagères et à l'isolement social ${ }^{12}$.

Les filles sont exclusivement soumises à la mutilation génitale, ablation forcée d'une partie de leur corps. Quelque 130 à 140 millions de filles et de femmes ont subi cette mutilation et trois millions de fillettes en courent le risque chaque année ${ }^{13}$. Outre une violation profonde de leurs droits humains, la mutilation génitale des filles représente un grave danger pour leur santé, donnant souvent lieu à des situations d'hémorragie, d'infection, de rapports sexuels douloureux, de lésion de l'appareil urinaire, de cicatrices et d'autres incapacités graves et durables. Elle accroît par ailleurs les risques de la grossesse et de l'accouchement et est associée à de faibles résultats de naissance.

Le mariage précoce est un autre exemple de pratique traditionnelle néfaste infligée disproportionnément aux filles. Les adolescentes sont beaucoup plus susceptibles que leurs homologues masculins d'être mariées et soumises de force à l'activité sexuelle pendant l'enfance. Dans le monde en développement (hors Chine), environ un tiers des adolescentes sont mariées avant l'âge de 18 ans. Dans certains pays, près de $30 \%$ le sont avant l'âge de 15 ans $^{14}$. Les filles les plus pauvres, qui courent le plus grand risque d'être mariées très jeunes, sont les plus vulnérables au VIH et aux infections sexuellement transmissibles (IST), à la maternité prématurée et fréquente, aux
PROBLÈMES ÉMERGENTS POUR LES FILLES DÈS L'ÂGE DE 12 ANS*

Maturation sexuelle

Consolidation des normes de genre

Fardeau disproportionné des soins et du travail

Exclusion des espaces publics et manque de sécurité dans ceux accessibles

Sécurité scolaire et déscolarisation

Perte des pairs

Pression du mariage précoce

Pratiques traditionnelles néfastes

Migration à la recherche de travail

Soumission aux médias sexualisés et de consommation

*Bruce, J. 2010. Investing in Adolescent Girls: Building the Health, Social, and Economic Assets of the Poorest Girls in the Poorest Communities. Présentation DFID, Londres.

taux élevés de morbidité et de mortalité maternelle et aux niveaux supérieurs de mortalité et de mauvaise santé infantile ${ }^{15}$.

Dans la plupart des régions du monde, les filles deviennent sexuellement actives vers la fin de l'adolescence, entre les âges de 15 et 19 ans. Le papillomavirus humain (HPV) affecte les femmes relativement jeunes, dont près de $90 \%$ dans les pays en développement. II représente la cause principale d'années de vie perdues au cancer dans le monde en développement. Dans les contextes où la vaccination anti-HPV est disponible, les filles doivent en bénéficier avant le début de l'activité sexuelle ${ }^{16}$. L'activité sexuelle précoce des filles est le plus souvent associée à la contrainte et à la violence. Plus la femme est jeune au moment de ses premiers rapports sexuels, plus il y 
a de chances qu'elle y ait été forcée ${ }^{17}$. L'activité sexuelle précoce, par viol ou inceste en particulier, s'accompagne de diverses implications de santé, de la grossesse non désirée et des IST aux séquelles psychologiques et physiques durables. Les filles victimes de la violence sexuelle - et elles sont nombreuses - nécessitent une réponse immédiate et efficace du système sanitaire, avec contraception d'urgence (CU), prophylaxie post-exposition au VIH (PPE) et services de santé mentale et d'assistance juridique ${ }^{18}$.

Les données font état d'une faible pratique contraceptive parmi les jeunes femmes à l'occasion de leurs premiers rapports sexuels ${ }^{19}$. L'accès des adolescentes à la contraception est limité par leur propre manque d'information et de compétences, mais aussi par le fait que la plupart des services de santé génésique du monde ne sont pas conçus pour les atteindre ou répondre à leurs besoins. Dès le moment où elles deviennent sexuellement actives, elles devraient pourtant bénéficier d'un accès fiable à la contraception et de services appropriés de dépistage régulier des IST et de suivi de leur santé génésique.

Pour les adolescentes pauvres, les implications de la grossesse sont souvent sombres. Les complications liées à la grossesse et à l'accouchement comptent au nombre des causes principales de mortalité parmi les jeunes de 15 à 19 ans dans les pays en développement ${ }^{20}$. Ajoutée à leur faible rang social, la grossesse mène souvent à une déficience nutritive (telle que l'anémie), qui contribue à son tour au risque de fausse-couche, de mortinatalité, d'accouchement prématuré, de mortalité périnatale, de risque accru de transmission verticale du VIH et de fistule obstétricale, en particulier chez les mères primipares les plus jeunes ${ }^{21}$.

Parce que de nombreuses adolescentes se trouvent confrontées à une grossesse non désirée - produit, souvent, de rapports sexuels imposés par la force ou par la contrainte --, les taux d'avortement non médicalisé qui les concernent sont élevés. D'après les données disponibles pour l'Afrique, les filles âgées de 15 à 19 ans représentent $25 \%$ des IVG non médicalisées ${ }^{22}$.
Sous l'effet d'une combinaison de facteurs biologiques et sociaux (notamment la violence sexuelle et le viol, les rapports sexuels avec des hommes plus âgés, les échanges de faveurs sexuelles contre argent et produits de première nécessité, l'information limitée, la difficulté de négociation de l'usage du préservatif et d'accès aux services de santé), les jeunes femmes sont particulièrement vulnérables à l'infection à $\mathrm{VIH}$, surtout en Afrique subsaharienne où l'épidémie fait rage ${ }^{23}$. Dans l'ensemble, les filles sont plus susceptibles que les garçons de subir toutes les formes de harcèlement et d'abus sexuels, bien que la sous-déclaration par les deux sexes demeure problématique ${ }^{24}$.

Les filles pauvres portent aussi une charge distincte et souvent plus lourde que les garçons en ce qui concerne les tâches domestiques. II en résulte de plus hauts taux d'accidents, tels que chutes et brûlures, ainsi qu'une exposition supérieure aux toxines émanant des matériaux et des techniques ménagères, de cuisine notamment ${ }^{25}$. Nombre de filles issues des communautés pauvres n'ont d'autre choix que de migrer, en quête de meilleures possibilités d'instruction ou d'emploi, ou pour fuir un mariage précoce forcé ou d'autres circonstances familiales difficiles. Du milliard de travailleurs migrants dénombrés dans le monde, $72 \%$ sont de sexe féminin. Ces travailleurs sont souvent sans papiers ; ils gagnent généralement moins, doivent accepter des emplois exigeants et dangereux et sont souvent vendus ou amenés par la ruse au commerce du sexe ${ }^{26}$. Manifestement distinctes et considérables, les implications sur la santé des filles migrantes exigent une réponse adaptée et ciblée du secteur sanitaire et d'autres intervenants.

Dans l'ensemble, les adolescentes pauvres subissent, du fait de leurs vulnérabilités sociales imputables à l'inégalité de genre, à la discrimination et à la pauvreté, une charge-santé particulière et disproportionnée $e^{27}$. Le désavantage social des filles par rapport aux garçons et aux hommes est la cause profonde de la plupart de ces vulnérabilités. Notamment :
- Ia déscolarisation avant l'acquisition d'une éducation essentielle et de ses bienfaits sur la santé ;

- le mariage antérieur à l'accès des filles à leur pleine maturité physique et affective ;

- les premiers rapports sexuels forcés et les autres formes de violence ;

- les débouchés d'emploi limités, dans des conditions souvent dangereuses et sujettes à l'exploitation.

\section{Les comportements de santé} établis à l'adolescence peuvent affecter toute la vie, non seulement des filles et des femmes mais aussi des générations futures.

Les tendances comportementales qui affectent la santé, comme le régime alimentaire et l'activité physique, s'établissent souvent à l'adolescence. Les mauvaises habitudes peuvent conduire à la surcharge pondérale et à l'obésité. Les adolescentes obèses tendent à le rester à l'âge adulte et à accroître ainsi leur risque de diabète et de maladie cardiovasculaire. Si les adolescentes de nombreux pays souffrent toujours de dénutrition, les données de 20 pays à revenu faible ou intermédiaire font état d'une surcharge pondérale affectant quelque $12 \%$ des filles scolarisées de 13 à 15 ans $^{28}$. Malgré les avantages connus de l'activité physique sur la santé physique et mentale, les données de 36 pays à revenu faible ou intermédiaire révèlent que $86 \%$ des filles n'atteignent pas les niveaux d'activité physique recommandés, soit une proportion largement supérieure à celle observée chez les garçons.

L'adolescence est une période naturelle de curiosité et d'expérimentation émergentes, mais la consommation et l'abus prolongés de substances toxiques peuvent avoir des conséquences néfastes sur la santé. Les adolescentes fument et boivent de plus en plus, tandis que les données indiquent un ciblage grandissant des filles et des femmes dans la publicité du tabac ${ }^{29}$.

Le Tableau 2 souligne les conditions et problèmes de santé rencontrés par les adolescentes, leurs conséquences 


\section{TABLEAU 2 RISQUES ET BESOINS DE SANTÉ DES ADOLESCENTES VULNÉRABLES}

\section{PROBLÉMATIQUE SANTÉ DES ADOLESCENTES LA SANTÉ}

Malnutrition et dénutrition

Changements physiques et affectifs de la puberté

Menstruation

Déscolarisation

Mariage d'enfants

Grossesse précoce

Pratiques traditionnelles néfastes, notamment la mutilation génitale des filles

Violence physique et sexuelle
Anémie ; chétivité ; déficits cognitifs ; transmission intergénérationnelle.

Gêne physique, confusion affective ; dépression ; retrait social.

Gêne physique ; embarras ; retrait social.

Accès limité à l'éducation sanitaire et aux services de santé ; isolement et retrait social ; dépression ; charge de travail supérieure et risque accru d'accidents et d'exploitation ; pression de mariage précoce.

Le mariage d'enfants se pratique avant l'âge légal de consentement et peut mener à la violence et aux abus physiques et sexuels; isolement et retrait social ; grossesses précoces ; risque de contraction du VIH et d'autres IST.

La grossesse précoce peut être cause de morbidité (fistule obstétricale, par exemple) et de mortalité maternelle, de morbidité et mortalité infantile et d'avortement non médicalisé.

Douleur physique ; infection ; morbidité et mortalité à brève, moyenne et longue échéance.

Morbidité et mortalité à brève et à longue échéance ; dépression et suicide.

\section{SOLUTIONS NÉCESSAIRES POUR ATTÉNUER LE RISQUE}

Accès à l'information sur la santé ; accès égal et constant à des sources d'alimentation nourrissantes et équilibrées ; compléments alimentaires et vitamines.

Accès à une information complète et précise et accompagnement social ; espace privé et fournitures nécessaires à l'hygiène personnelle; espace sûr où rencontrer pairs et mentors et apprendre les compétences de vie.

Connaissance des fonctions génitales et de l'hygiène féminines ; accès à des fournitures de gestion de la menstruation (serviettes, tampons, médication antiinflammatoire non stéroïdienne) ; accès à un espace privé et à l'eau propre au domicile, à l'école et au travail.

Connaissance du droit à l'éducation et aux soins de santé ; accès à une éducation à la sexualité de qualité et aux services de santé à travers l'école ; espace sûr où rencontrer pairs et mentors et apprendre les compétences de vie.

Connaissance du droit à l'âge légal du mariage ; compétences et pouvoir de négocier l'âge désiré du mariage; compétences et pouvoir de négocier des rapports sexuels consensuels et sans risques; accès à l'information et aux services de santé ; espace sûr où rencontrer pairs et mentors et apprendre les compétences de vie.

Accès à l'information sur la santé, à la contraception et à de larges services de santé sexuelle et génésique et conseil sur les options possibles ; espace sûr où rencontrer pairs et mentors et apprendre les compétences de vie.

Connaissance du droit à l'intégrité corporelle ; compétences et pouvoir de négocier ; accès à l'information et aux services de santé ; espace sûr où rencontrer pairs et mentors et apprendre les compétences de vie.

Connaissance de ses droits ; promotion de normes de genre équitables; accès immédiat aux services juridiques, de santé et sociaux ; accès à la CU, à la PPE et autres services d'urgence en cas de viol ; dépistage et traitement du VIH/sida ; compétences et pouvoir de négocier la sécurité et le refuge ; espace sûr où rencontrer pairs et mentors et apprendre les compétences de vie ; plan de sécurité préventif. 


\section{TABLEAU 2 (SUITE)}

\section{PROBLÉMATIQUE SANTÉ DES ADOLESCENTES \\ CONSÉQUENCES POTENTIELLES SUR LA SANTÉ}

Isolement social ; mobilité et autonomie réduites ; discrimination et violence de genre accrues.

normes de genre

restrictives

Obésité

Gêne physique ; dépression ; retrait social ; hypertension et diabète précoces.

Tabac, drogue et alcool

Altération du jugement corrélée à des risques, accidents et lésions de SSG accrus ; affections de santé chroniques.

Dépression et troubles de santé mentale

Fonctionnement sous-optimal ; retrait social ; troubles de l'alimentation ; suicide.

Travail domestique et autre dangereux avec risque d'exploitation
Épuisement physique ; accidents et blessures; sévices psychologiques, physiques et sexuels ; dépression et anxiété.

\section{SOLUTIONS NÉCESSAIRES POUR ATTÉNUER LE RISQUE}

Connaissance de ses droits ; promotion de normes de genre équitables ; accès aux services sociaux ; accompagnement social ; espace sûr où rencontrer pairs et mentors et apprendre les compétences de vie.

Accès à l'information, aux ressources et à l'accompagnement social pour une bonne alimentation ; accès à une alimentation saine et abordable ; espaces sûrs où pratiquer des activités physiques et sportives et où rencontrer pairs et mentors et apprendre les compétences de vie.

Connaissance et information sur les risques et les signes de la dépendance ; encadrement social et par les pairs.

Connaissance et information sur les symptômes ; encadrement social et professionnel.

Connaissance de l'âge légal du travail ; compétences et pouvoir de négocier la sécurité et une rémunération équitable ; accès à l'information et aux services de santé ; espace sûr où rencontrer pairs et mentors et apprendre les compétences de vie. potentielles sur leur santé et les solutions possibles pour amoindrir le risque.

\section{Les systèmes de santé ne} répondent pas aux besoins des adolescentes vulnérables - en tant que consommatrices ou que prestataires de soins et services.

Le secteur de la santé joue un rôle essentiel à la protection et à l'assurance de la santé des adolescentes. L'accès aux filles les plus pauvres des communautés les plus démunies exige cependant d'aller au-delà de l'enceinte clinique traditionnelle. Par définition, les sous-groupes de filles marginalisés sont difficiles à identifier et à joindre. II est dès lors crucial de savoir où ils se trouvent et d'aller à leur rencontre ${ }^{30}$. L'émergence de "services de santé favorables aux jeunes " (SSFJ) repré- sente un pas important dans la bonne direction : ces services s'efforcent d'offrir plus de pertinence et un meilleur accès aux jeunes en leur proposant une gamme complète de prestations, en prolongeant leurs heures d'ouverture, en leur assurant confidentialité et respect de la vie privée, en rendant leurs installations plus accueillantes, en formant leur personnel à servir efficacement les adolescents et en amoindrissant l'obstacle des coûts. L'OMS a mis au point une documentation de travail utile aux SSFJ31,32,33 et de nombreuses organisations y ont contribué leurs outils et guides. Les responsables de la politique internationale et les gouvernements nationaux définissent davantage les normes et les directives, mais la mise en œuvre reste inégale et n'atteint pas souvent les filles pauvres.
II existe un besoin continu d'apport d'information et de services de santé essentiels aux sous-groupes de filles vulnérables là où elles peuvent être identifiées : tôt, avant que ces filles ne quittent l'école, au sein des communautés, sur leurs lieux de travail et à travers les institutions et établissements autres que de santé où elles sont plus susceptibles de se rassembler (marchés, magasins/kiosques de quartier, lieux de culte, ONG et organisations communautaires). D'autres approches et stratégies seront aussi requises pour atteindre ces filles de manière appropriée et pour améliorer leur santé et leur sécurité : par approche et distribution communautaire de produits sanitaires, services de santé mobiles, marketing social et usage des technologies émergentes telles que les téléphones cellulaires. Si les filles 
peuvent et doivent jouer un rôle dans la prestation formelle de services de santé en établissement, les efforts déployés pour les atteindre peuvent être particulièrement créatifs et efficaces au niveau local.

En tant que prestataires et soignantes formelles et informelles au sein du système sanitaire et au niveau de la famille et de la communauté, les filles et les jeunes femmes apportent d'ores et déjà une contribution majeure à la prestation de soins de santé. Malgré son rôle largement disproportionné aux niveaux primaires, le sexe féminin n'est guère représenté aux rangs supérieurs et reste généralement concentré aux niveaux d'emploi moins bien rémunérés et exposés aux plus grands risques professionnels. Dans leurs rôles de prestataires de soins informelles au foyer et dans la communauté, les filles et les jeunes femmes ne sont pas souvent soutenues, reconnues ou rémunérées ${ }^{34}$. II peut être doublement bénéfique de renverser la situation et de former, soutenir et rémunérer les jeunes femmes pour qu'elles entrent dans le secteur de la santé. Certaines filles, même dans les communautés les plus pauvres, ont atteint ainsi un niveau d'éducation plus avancé. Ces femmes peuvent du reste aider à combler les insuffisances de ressources humaines et apporter une information et des services de santé de qualité aux sous-populations même dont elles sont membres. L'effort peut tout à la fois étendre la portée du secteur de la santé et susciter un mode de subsistance viable pour les jeunes femmes.

\section{POLITIQUES ET PRATIQUES PRÉCONISÉES}

Mener une recherche ciblée sur les problèmes de santé particuliers ou partagés des adolescentes vulnérables

Même si les adolescentes sont biologiquement et socialement différentes de leurs homologues masculins, les études et la recherche cliniques menées dans le monde portent principalement sur des sujets adultes de sexe mascu- lin. Cette approche suscite de vastes lacunes de données scientifiques en ce qui concerne le moment et le mode d'impact de différentes maladies sur le sexe féminin et, principalement, la manière dont les protocoles thérapeutiques devraient peut-être varier en fonction de l'âge et du sexe. Les études ont observé que les fardeaux représentés par le jeune âge et la féminité alourdissent les risques biologiques et sociaux et les conséquences vécues par les adolescentes. Celles-ci sont par exemple particulièrement vulnérables au paludisme. Dans de nombreux contextes d'Afrique subsaharienne, les adolescentes sont souvent parasitémiques et anémiques dès le début de la grossesse. D'après les données du Malawi, les adolescentes, enceintes ou non, présentent des niveaux parasitaires significativement supérieurs à ceux des femmes âgées de plus de $19 \mathrm{ans}^{35}$. Des protocoles spécifiques au sexe et à l'âge sont par conséquent nécessaires au niveau de la recherche étiologique et de l'éclaircissement des éléments sexospécifiques des maladies infectieuses et chroniques des filles et des jeunes femmes. S'il est établi que les filles et les femmes sont souvent traitées plus tard et moins agressivement que leurs homologues de sexe masculin ${ }^{36}$, les implications sanitaires, sociales et financières du déséquilibre restent obscures.

\section{Cartographier l'état de santé} des filles et leurs expériences sociales étroitement liées pour comprendre les vulnérabilités spécifiques à l'âge, au genre et au contexte des sous-groupes de filles et jeunes femmes.

Les données nécessaires à la cartographie des vulnérabilités démographiques et de santé des groupes d'adolescentes existent. Grâce à ces données, on pourrait déterminer où les investissements - de politique et programmatiques ou monétaires et de ressources - sont les plus urgents et les plus critiques. La cartographie peut aussi servir de guide aux décisions d'investissement dans l'infrastructure des soins de santé. Ainsi, elle peut aider à identifier les besoins les plus intenses de soutien communautaire - la prévention des grossesses non désirées et l'accompagnement des grossesses d'adolescentes à risques, par exemple. L'expérience programmatique, au Guatemala et ailleurs, montre que les adolescentes elles-mêmes peuvent participer aux exercices de cartographie. Les filles peuvent incorporer leurs perspectives de la santé et de la sécurité dans les efforts de cartographie et partager leurs idées sur la manière d'améliorer l'accès aux services de santé et leur qualité. L'approche peut s'avérer particulièrement utile aux initiatives de prévention de la violence de genre. Les filles peuvent montrer et expliquer au personnel responsable du programme où et quand elles se sentent en sécurité ou non dans leurs communautés et leurs quartiers. La collaboration avec les filles permet de concevoir des programmes adaptés au contexte qui réduisent le risque et renforcent la sécurité. Ces programmes peuvent inclure des stratégies de protection des filles et des jeunes femmes, tout en assurant la promotion de normes de genre plus équitables ${ }^{37}$.

Mener une recherche participative et tester les stratégies pour mieux comprendre et soutenir le double rôle des filles et des jeunes femmes en tant que consommatrices et prestataires de soins de santé.

II importe d'identifier les connaissances et les compétences dont les filles et les jeunes femmes estiment avoir besoin pour créer un système de santé plus équitable, plus sensible et plus efficace. On maximiserait ainsi leur apport plutôt que de verser dans l'exploitation et la discrimination. Un atelier récent mené au Guatemala a mis en évidence l'énergie et l'intelligence avec lesquelles les filles décrivent leurs besoins interconnectés $^{38}$. La liste ci-dessous énonce quelques exemples des types de 
ressources sanitaires, sociales et économiques que les adolescentes jugent nécessaires :

- Connaissance des signes et dangers de la violence de genre.

- Connaissance et capacité de prévention de la grossesse non planifiée.

- Capacité de réaction et de réponse à la violence interfamiliale (avoir un plan d'urgence).

- Connaissance des ressources de santé disponibles dans la communauté et ses alentours.

- Connaissance de l'endroit où aller pour obtenir de l'aide en cas d'urgence.

- Connaissance des personnes à qui s'adresser en cas de violences physiques ou sexuelles.

- Connaissance de leurs droits humains.

- Reconnaissance de leurs droits par les familles, les communautés et les écoles.

- Connaissance de la législation du travail.

- Capacité d'exiger la poursuite de la scolarisation.

- Possibilité de participation aux activités civiques.

- Connaissance de leur identité culturelle.

- Sens de l'estime de soi et de leurs propres capacités.

La programmation sensible qui apporte cette information aux filles et qui les dote des compétences et de l'accompagnement nécessaire à leur réalisation - pour elles-mêmes et pour les autres - peut jouer un rôle important dans la transformation des filles en " actif de santé " de leur propre vie et des communautés.

Atténuer les risques de santé que font courir aux adolescentes le manque de sécurité et la fréquence des menaces ou de l'expérience de la violence.

Presque partout dans le monde, les filles se trouvent chaque jour confron- tées à la menace de la violence - chez elles, dans l'espace public et à l'école. La violence de genre, l'exposition des filles à cette violence et son vécu dès un très jeune âge sont devenus la norme. Les adultes responsables tels que les enseignants, les agents sanitaires et la police ne sont pas suffisamment sensibilisés aux lois, aux politiques et aux systèmes de protection et de défense des filles et ne les appliquent donc pas. Les services sociaux et de santé sont généralement mal équipés pour prévenir la violence de genre et y réagir, particulièrement en ce qu'elle affecte les adolescentes. La réalité chronique insidieuse de cette violence limite, refoule et précipite les filles dans d'autres situations dangereuses : elles fuient parfois leur foyer, ou se suicident même ${ }^{39}$. Les filles choisissent souvent l'isolement social pour se protéger de la menace de la violence, se soustrayant davantage encore à l'information et aux services de santé, même minimes, existants. Une approche écologique est nécessaire si l'on veut renverser la situation. Des efforts doivent être déployés pour renforcer l'actif social, sanitaire et économique des filles vulnérables. De plus, les décideurs et les communautés doivent favoriser le changement des conditions des filles pour leur assurer plus de sécurité. On voit émerger différents efforts novateurs d'essai des stratégies de sécurité ${ }^{40}$, mais il reste beaucoup à accomplir pour identifier celles efficaces et les appliquer à plus grande échelle.

\section{Développer la capacité}

de réflexion critique des adolescentes concernant leur santé et leur vie.

Beaucoup des filles le plus en manque d'information et de compétences ne sont pas socialement connectées au réseau scolaire et autres prestataires. Les programmes qui cherchent véritablement à atteindre les plus pauvres des communautés les plus démunies doivent dès lors faire preuve d'imagination pour identifier et atteindre les segments concernés. Pour renforcer et élargir les compétences de vie et de réflexion critique des filles, de nouvelles approches pionnières de l'éducation à la santé et à la sexualité doivent être élaborées et mises en $œ u v r e$. Un seul programme ${ }^{41}$ offre un outil innovant d'enseignement et de transmission de ces compétences. II apporte aux filles une information de grande envergure, les ressources, l'accompagnement et le pouvoir qui leur permettent de prendre elles-mêmes les décisions qui concernent leur vie et leur corps.

Fournir aux médias des scénarios plus spécifiques concernant les risques posés à la santé des adolescentes et promouvoir une image positive et réaliste des filles.

Le déluge constant d'images médiatiques des filles et des femmes, partout dans le monde, est essentiellement contre-productif au message d'autonomisation et de présentation aux adolescentes de modèles de rôle normaux, sains et équilibrés. Au contraire, ces images déforment souvent grossièrement la réalité et renforcent les stéréotypes de genre négatifs, oppressifs et destructeurs. Elles minent la capacité des filles à développer une image saine de leur propre personne durant la transition de l'enfance à l'âge adulte. D'autres images positives et réalistes doivent être mises en valeur, comme celles présentées par Puntos de Encuentro ${ }^{42}$ au Nicaragua. L'expérience nicaraguayenne révèle combien les représentations médiatiques honnêtes et vraies, adaptées à l'âge de leur audience et sensibles aux questions de genre, d'orientation sexuelle et de race, peuvent être engageantes, informatives et propices au changement d'attitude.

Identifier et tester les possibilités de recours aux technologies de plus en plus accessibles pour améliorer la santé et la sécurité des filles.

Les filles des communautés les plus pauvres et les plus isolées du monde 
ont de plus en plus accès à l'information et à la technologie, qui peut et doit être explorée et testée comme instrument de promotion de la santé et de la sécurité. Une étude menée au Brésil a constaté que $84 \%$ des filles ont un téléphone mobile et $82 \%$ ont utilisé Internet ${ }^{43}$. Au Bangladesh, en Inde et en Afrique du Sud, l'initiative MAMA (Mobile Alliance for Maternal Action) a été lancée pour catalyser la puissance de la technologie mobile au profit de l'information des femmes enceintes. Celles-ci reçoivent une information par téléphone mobile sur l'hygiène de la grossesse, les mythes et les idées fausses, les signes de danger et l'accès aux services de santé locaux ${ }^{44}$. II reste à évaluer l'efficacité du programme et il n'existe pas encore beaucoup d'initiatives ciblées directement sur les adolescentes, mais les technologies de l'information et de la communication (TIC) n'en peuvent pas moins contribuer à l'apport opportun d'une information cruciale aux filles et les aider à établir la connexion les unes avec les autres et avec les réseaux et les services propices à leur santé, leur sécurité et leur bien-être.
Instaurer des programmes qui ciblent et soutiennent la transition critique de la puberté.

Les systèmes de santé du monde sont agencés pour considérer la situation des filles après que les pires résultats se sont déjà produits - y compris les rapports sexuels à risques et forcés et les grossesses précoces non désirées. Les adolescentes les plus vulnérables tendent à faire fausse route peu après la puberté. Aussi est-il essentiel d'engager plus tôt les programmes destinés à ces filles - dès l'âge de huit ou 10 ans - et de les atteindre à travers le réseau scolaire, quand la majorité d'entre elles peut être identifiée et jointe le plus aisément. Les approches telles que le " contrôle de bien-être " (voir le Tableau 3), proposé comme instrument de dépistage préventif socio-sanitaire global pour les filles et les garçons vers l'âge de la puberté, peuvent aider à garder les jeunes, en particulier les filles vulnérables, sur la bonne voie. De telles stratégies peuvent aussi veiller à ce que les filles restent en bonne santé et bénéficient d'une éducation suffisante et d'un accompagnement social approprié pour une bonne traversée de l'adolescence ${ }^{45}$.

Soutenir les mères primipares les plus jeunes, qui portent le fardeau d'une part disproportionnée et grandissante de la mauvaise santé maternelle.

Les filles mariées représentent la majorité des femmes sexuellement actives dans le monde en développement. Les programmes de jeunes et d'éducation à la sexualité, qui se concentrent principalement sur les jeunes célibataires scolarisés, ont cependant tendance à les omettre. Les programmes de santé génésique et de développement négligent aussi les filles mariées, en se concentrant davantage sur les femmes adultes. Les jeunes mères primipares souffrent souvent d'une faible mobilité sociale, d'un accès limité à l'information fiable et d'un pouvoir de décision génésique insuffisant. Elles sont souvent plus vulnérables à la violence que les femmes qui se marient à un âge plus avancé ; elles ne sont pas informées et ont des rapports non protégés avec des maris parfois beaucoup plus âgés

\section{TABLEAU 3 ÉLÉMENTS TYPES DE " CONTRÔLE DE BIEN-ÊTRE "}

\section{SANTÉ}

Visite médicale

Contrôle d'immunisation et rattrapage

Alimentation/contrôle de croissance

Information et services de santé sexuelle et génésique

Vaccin anti-HPV (si disponible)

Information sur la prévention du VIH/sida

Dépistage de la violence et accompagnement

Dépistage de santé mentale et accompagnement

Dépistage de blessures et prévention

\section{SOCIAL}

Conseil

Renforcement des compétences de vie

Évaluation d'ordre scolaire et accompagnement

Évaluation et amélioration du soutien par les pairs et de l'accompagnement social

Dépistage drogue/alcool/tabac et accompagnement préventif

Bien-être familial et accompagnement social

Renforcement de compétences et motivation à la citoyenneté et à la participation sociale 
qu'elles. Ces circonstances peuvent aggraver leur vulnérabilité au VIH et autres IST, limitant ainsi leur propre bien-être tout en accroissant le risque de mortalité maternelle et infantile. Les jeunes mères primipares doivent faire l'objet d'efforts programmatiques spécialement ciblés ${ }^{46}$.

En partant des filles, chercher à mieux comprendre et mesurer l'engagement positif des garçons et des jeunes hommes au soutien de la santé et du bien-être des filles vulnérables.

La portée de la programmation à l'intention propre des hommes et des garçons - en leur qualité d'époux, de petits amis, de frères et de pères - gagne du terrain. Les données indiquent combien les initiatives propices aux rôles et relations de genre équitables peuvent influencer favorablement les attitudes et les comportements déclarés par les garçons et les hommes $^{47}$. Les programmes commencent rarement par interroger les filles et les jeunes femmes sur les hommes présents dans leur vie (qui peuvent représenter à la fois protection et risque pour leur santé et leur bien-être), sur leur expérience et sur le changement qu'elles jugeraient utile. Cette information est nécessaire à la conception d'interventions qui impliquent spécifiquement les garçons et les hommes qui impactent la vie des filles et des jeunes femmes. La mesure d'efficacité des interventions en termes d'amélioration des relations de genre et d'engagement masculin positif doit être entreprise avec les participants de sexe masculin, certes, mais surtout avec les filles et les femmes qui partagent leur vie ${ }^{48}$.

Mobiliser les filles et les jeunes femmes pour renforcer le secteur de la santé et élargir leurs horizons de subsistance.

D'un point de vue historique et culturel, les filles et les jeunes femmes assument largement les soins des malades au sein des familles et des communautés (elles soignent les enfants malades, les parents, les grands-parents et les personnes vivant avec le VIH et d'autres maladies chroniques). La responsabilité disproportionnée de ces soins peut entraver la scolarisation des filles et leur aptitude à l'emploi en dehors du foyer, ainsi que leur poursuite et réalisation maximale de leur potentiel. II importe de mieux cerner les dimensions, les implications et le coût de la véritable charge des soins familiaux et communautaires qui pèsent sur les filles et les jeunes femmes, ainsi que la manière dont la situation pourrait être rendue plus équitable.

Un programme mené avec les filles mayas indigènes du Guatemala a illustré l'engagement et le travail des jeunes femmes dans le secteur informel de la santé, au titre de guérisseuses et sages-femmes traditionnelles ${ }^{49}$ Les jeunes femmes qui vivent dans la pauvreté sont toujours en quête d'options de subsistance sûres et dignes, notamment dans le secteur de la santé. L'effort n'est cependant pas sans obstacles, en particulier pour celles des communautés les plus pauvres où les services sanitaires font le plus défaut. II serait possible de changer les choses, mais seulement dans la mesure où le secteur de la santé peut trouver le moyen de former les jeunes femmes, de les soutenir et de leur procurer un emploi sûr, digne et légitime, où leurs connaissances et leurs compétences trouvent rémunération. Souvent, les filles les plus vulnérables qui aboutissent dans les rôles de soignantes familiales et communautaires informelles se retrouvent dans cette situation parce que le secteur formel de la santé n'atteint pas le milieu où elles vivent. Une stratégie efficace pourrait faire des jeunes femmes compétentes, motivées - et souvent multiculturelles et multilingues - des travailleuses de proximité ou agents sanitaires formelles au niveau communautaire. Les jeunes femmes déjà actives au titre de guérisseuses et sages-femmes traditionnelles peuvent être intégrées formellement dans le secteur de la santé. Elles peuvent aider à combler le manque de ressources humaines dans les communautés rurales ou sous-desservies.

Les jeunes femmes peuvent aussi être formées et déployées pour aider à répondre aux besoins de santé des communautés et des filles, au soutien notamment du secteur sanitaire formel de niveau primaire, qui atteint les plus vulnérables. Des efforts d'intégration des jeunes dans le secteur de la santé au titre de pairs éducateurs et de conseillers ont été entrepris dans plusieurs pays ${ }^{50}$. Les jeunes femmes peuvent aussi être formées à :

- entreprendre la distribution et le marketing social communautaire des technologies sanitaires utiles à l'ensemble de la communauté (filtres à eau, dispositifs de cuisine sanitaires et moustiquaires), ainsi que des fournitures de santé sexuelle et génésique (comme le préservatif féminin et masculin) ;

- guider et soutenir la prestation de services de santé mobiles dans leur communauté ;

- apporter en d'autres emplacements locaux hors secteur sanitaire (dans les écoles, les pharmacies, sur les marchés, dans les organisations communautaires, les lieux de culte et dans le cadre des programmes d'espaces sûrs pour les filles) une information et des services de santé adaptés à l'âge, au genre et à la culture de leur audience.

D'autres exemples de promotion communautaire de compétences vitales pour les filles sont énoncés au Tableau 4.

II est essentiel que les jeunes femmes soient rémunérées équitablement et soutenues efficacement dans leurs nouveaux rôles d'accompagnement et d'amélioration de la portée et de la qualité du secteur de la santé à ces différents niveaux.

De nouvelles expériences basées sur les stages professionnels pour 


\section{TABLEAU 4 COMPÉTENCES ESSENTIELLES DE PROMOTION DE LA SANTÉ COMMUNAUTAIRE DES FILLES}

\section{AVANT L'ÂGE DE 10 ANS}

Sait comment se laver convenablement les mains.

Sait le nom de l'agent sanitaire de proximité et de l'infirmière et du médecin locaux.

Sait comment se rendre au centre de santé et à l'hôpital le plus proche.

Sait comment demander un transport d'urgence.

Sait reconnaître les signes de maladie diarrhéique chez le nourrisson et l'adulte.

Sait reconnaître les signes de danger pendant le travail et l'accouchement.
AVANT L'ÂGE DE 14 ANS

Sait comment gérer la menstruation et l'hygiène féminine.

Sait comment fonctionnent l'appareil génital masculin et l'appareil génital féminin.

Sait comment prévenir une grossesse non désirée.

Sait comment se transmet le VIH.

Sait comment utiliser le préservatif.

Sait où obtenir un test de dépistage du VIH.

Sait reconnaître les situations de violence de genre et y réagir (a un plan d'urgence).

A reçu une éducation complète à la sexualité. donner aux filles l'occasion de participer à différents programmes de santé et d'accompagnement social font leur apparition au niveau communautaire, dans le secteur public et dans les communautés locales. Au Guatemala, de jeunes stagiaires indigènes sont formées dans le secteur public à entreprendre des activités de prévention de la violence et à apporter aux victimes de viols, souvent très jeunes, une information et un soutien bilingues adaptés à leur culture ${ }^{51}$.

Un effort concerté doit être déployé pour valoriser davantage les jeunes femmes au titre d'agents sanitaires. En comparant les vulnérabilités de santé des filles d'un endroit particulier avec la disponibilité de celles dotées d'un niveau d'instruction élémentaire - et donc susceptibles de devenir les agents sanitaires du changement -, les décideurs politiques et les responsables des programmes peuvent identifier les meilleurs emplacements de leurs premiers investissements. Nous avons beaucoup à apprendre des filles et leurs perspectives peuvent nous guider quant aux meilleurs moyens de soulager leur fardeau et de maximiser leur potentiel.

Informer les filles et les jeunes femmes pour qu'elles comprennent leurs droits et leurs responsabilités en leur qualité de consommatrices comme de prestataires de soins de santé.

Toutes les filles, en particulier les plus vulnérables, doivent savoir qu'elles ont droit à une information, une éducation, un conseil et des services de santé complets, exacts et pertinents. Outre cette sensibilisation pour leur propre gouverne, beaucoup de filles et de jeunes femmes peuvent être formées à la prestation de ces services au niveau de la communauté (dans le secteur de la santé public comme privé), où elles pourront ainsi trouver un moyen de subsistance sûr, productif et socialement responsable. Les jeunes femmes se trouvent souvent en position unique pour servir de liaison biculturelle bilingue entre leurs familles et communautés et le secteur de la santé, en tirant du reste parti d'un rôle qu'elles assument déjà. La mobilisation et le soutien des jeunes femmes au titre d'agents sanitaires représentent une approche gagnante pour les filles comme pour le secteur de la santé.

\section{RESSOURCES : PROGRAMMES " LABORATOIRES D'APPRENTISSAGE ", PUBLICATIONS ET OUTILS}

Abriendo Oportunidades : Travailler avec les filles du Guatemala rural pour découvrir leur potentiel de santé et réduire la violence.

Le programme Abriendo Oportunidades (AO) du Population Council est conçu pour atteindre et autonomiser les filles indigènes de 8 à 18 ans du Guatemala rural. Le programme repose sur le modèle des " espaces sûrs " pour donner aux filles l'occasion de se rassembler régulièrement et élargir ainsi leur réseau social, leur apprendre les compétences de vie, les aider à poursuivre leur scolarisation, faciliter 
leur transition vers des moyens de subsistance dénués de risques et leur donner accès à des modèles de rôle et mentors féminins. Le programme a entrepris l'expérimentation des espaces sûrs en tant que plates-formes de formation de leaders féminines aptes à assurer l'éducation sanitaire et quelques services de santé dans les communautés. II s'agit là d'une stratégie à tester et évaluer.

En collaboration avec une ONG locale de micro-consignation, AO entend aussi former des filles appelées à devenir les leaders de campagnes de sensibilisation et à vendre des produits de santé communautaire (filtres à eau, moustiquaires, lunettes de lecture, etc.) dans leurs communautés rurales, en gardant un petit bénéfice pour elles-mêmes. Pour plus d'information, s'adresser au consultant du Population Council Angel del Valle (adelvalle@ popcouncil.org) ou voir le site Web du projet sur http://www.popcouncil. org/projects/244_CreateOpportunities Mayan.asp.

Un effort concerté doit être déployé pour valoriser davantage les jeunes femmes au titre d'agents sanitaires.

Rwanda 12+ : Concentrer une attention systématique sur le potentiel et les besoins de la fille de 12 ans.

Au Rwanda, les ministères de la Santé, de la Jeunesse et de l'Éducation ont entrepris un projet de collaboration avec le Population Council, le Girl Hub du DFID et la Nike Foundation, pour l'expérimentation du concept " 12+ check-in " par lequel différents services de santé et de bien-être social sont offerts aux filles - visite médicale, contrôle d'immunisation et rattrapage, dépistage de la violence et soutien, évaluation scolaire et renforcement des compétences de citoyenneté -, programmés pour les atteindre au moment critique de la puberté. L'initiative a pour intention d'aider les filles à rester ou à s'engager sur la bonne voie en leur offrant une évaluation complète de leurs besoins et en les connectant ainsi que leur famille aux services sociaux existants. L'initiative donnera l'occasion d'identifier les erreurs de trajectoire et d'y remédier concernant les conditions souvent cachées des adolescentes au-delà des chiffres globaux. Les données démographiques et de santé peuvent être collectées et suivies pour permettre l'évaluation longitudinale de la santé et du bien-être féminins, avec groupement d'un sous-ensemble des filles aux âges critiques. Pour plus d'information, s'adresser à Janna McDougall (Janna.McDougall@ nike.com).

\section{Berhane Hewan : Créer un espace de soutien des filles non mariées et mariées et des plus jeunes mères primipares.}

Dans la région éthiopienne d'Amhara, le mariage et l'activité sexuelle précoces vont de pair. Vingt-six pour cent des filles mariées ont connu leurs premiers rapports sexuels avant l'âge de 12 ans, et $70 \%$ avant l'âge de 15 ans. Moins du tiers (31\%) des filles mariées avaient commencé à avoir leurs règles au moment de leurs premiers rapports. Quatre-vingt-deux pour cent n'avaient pas désiré ces premiers rapports et $81 \%$ les ont qualifiés de forcés. Les chiffres sont encore plus élevés parmi les filles mariées plus jeunes encore. D'autres données qualitatives mettent en évidence les problèmes de violence et de non-consentement.

Pour y faire face, le programme Berhane Hewan, mis en œuvre par le Population Council en collaboration avec le ministère éthiopien de la Jeunesse et des Sports, entre en contact avec les filles mariées ou non de 10 à 19 ans et établit des mécanismes pertinents et efficaces de protection et de soutien des filles vulnérables au mariage précoce et des adolescentes mariées. Les filles mariées - de 11 à 20 ans - se réunissent au moins une fois par semaine, le dimanche aprèsmidi, pendant plusieurs heures. Elles sont informées sur leur santé et celle de leurs enfants à venir (beaucoup sont déjà enceintes). Elles sont aussi sensibilisées et bénéficient d'un accès aux méthodes de planification familiale et elles développent leur plan de sécurité autour de leur grossesse. Le soutien apporté par ces clubs s'est traduit par un taux de pratique contraceptive de $74 \%$ parmi les filles mariées incluses dans le programme, par rapport à $45 \%$ parmi celles d'un village témoin. On observe aussi une augmentation de la connaissance spécifique des risques de contraction du VIH au sein du mariage et une augmentation de la proportion des femmes et de leurs partenaires qui se soumettent au dépistage du $\mathrm{VIH}_{52}$. Pour plus d'information, s'adresser à la directrice nationale du Population Council en Éthiopie, Annabel Erulkar (aerulkar@ popcouncil.org) ou voir le site Web du projet sur http:// www.popcouncil.org/projects/100_ berhaneHewanEthiopia.asp.

\section{Puntos de Encuentro : Au carre- four de la violence et de la santé précaire.}

Puntos de Encuentro est une ONG locale nicaraguayenne dont la mission est d'accroître la capacité des femmes et des jeunes à prendre le contrôle de leur propre vie et à participer à tous les niveaux de la société. Pour accomplir cette mission, Puntos de Encuentro met en œuvre une stratégie multimédiatique et multiméthodes combinant des programmes d'"éduanimation ", de mobilisation sociale et de renforcement des capacités locales pour encourager le changement comportemental individuel, la communication interpersonnelle, le soutien social et l'action collective. Un échantillon de plus de 4500 jeunes hommes et femmes âgés de 13 à 24 ans a été soumis aux mesures quantitatives et qualitatives d'une évaluation d'impact. 
Les résultats font état d'une réduction significative de l'opprobre et des attitudes de genre inéquitables, d'une connaissance et d'un recours accrus aux services relatifs au VIH et d'une amélioration significative de la communication interpersonnelle sur la prévention du VIH et le comportement sexuel. Les observations qualitatives révèlent aussi le rôle important du programme dans la promotion du dialogue communautaire, le renforcement du leadership des jeunes et l'encouragement des alliances avec d'autres organisations. Les résultats laissent de plus entendre que les comportements individuels (tels que l'usage constant du préservatif dans les relations sexuelles) ne sont pas isolés de l'environnement dans lequel les gens vivent mais qu'ils sont plutôt liés aux contextes et aux processus sociaux. Pour plus d'information, s'adresser à Amy bank (amy.bank@ puntos.org.ni) ou télécharger la description du programme depuis http://www. endvawnow.org/pampa/v0.1/library/ filemanager/v1/files/Sexto\%20Sentido.pdf.

\section{Outils de travail concernant les filles et la santé}

Un seul programme : Guide pour une approche pédagogique unifiée de la sexualité, du genre, du VIH et des droits humains.

Un seul programme est conçu pour aider les enseignants, les formateurs et les jeunes leaders à développer un programme d'éducation concernant la sexualité et le VIH axé sur le genre et les droits. Le but est d'aider les éducateurs à aborder avec les jeunes les questions des normes de genre, de la communication et de la prise de décision, du consentement sexuel et de la contrainte, de l'équité et des droits humains (y compris les droits sexuels), du pouvoir et des relations, de la prévention du VIH, des IST et des grossesses non planifiées, de la puberté et du changement social. Les directives sont innovantes dans leur intégration thématique, leur accent sur les méthodes d'enseignement expérientiel dynamique et leur objectif d'enseignement propice au développement de la réflexion critique chez l'adolescent. Les deux volumes unissent la matière au cœur du programme et les activités participatives proposées pour les enseigner, y ajoutant une section de ressources complémentaires. La matière et l'approche peuvent être adaptées et évaluées en fonction de différents sous-groupes de filles et de jeunes femmes dans différents contextes. L'ouvrage peut être téléchargé gratuitement en anglais, en français et en espagnol depuis http://www. itsallone.org.

Investing When It Counts: Generating the Evidence Base for Policies and Programs for Very Young Adolescents.

Cette publication offre un guide aux responsables politiques et programmatiques sur les considérations techniques et éthiques de la recherche et de la conception programmatique relatives aux très jeunes adolescents, en particulier les filles âgées de 10 à 14 ans. Elle peut être téléchargée gratuitement en anglais et en espagnol depuis http:// www.popcouncil.org/pdfs/InvestingWhenltCounts.pdf.

Guide pratique de l'OMS pour les soins aux adolescents : Un outil de référence destiné aux agents de santé de premier niveau.

Ce guide procure un outil de référence aux agents de santé (médecins, personnels infirmiers et cliniques formés et agréés) chargés de la prestation de services aux enfants, aux adolescents et aux adultes. Son objectif est de les aider à soigner leurs patients adolescents de manière plus efficace et plus sensible. II s'agit d'un guide pratique détaillé et précis sur la manière d'aborder et de traiter les adolescents qui leur présentent un problème ou une préoccupation concernant leur santé ou leur développement. L'ouvrage peut être téléchargé gratuitement depuis http://www.who.int/maternal_ child_adolescent/documents/ 9789241599962/en/index.html.
Conception d'un programme centré sur les filles : Boîte à outils pour l'élaboration, le renforcement et l'expansion de programmes destinés aux adolescentes.

Cette " boîte à outils " s'adresse à toute personne désireuse de travailler avec des adolescentes de 10 à 24 ans. Elle peut être utile au concepteur ou à l'exécutant d'un programme, à la personne chargée de la rédaction d'une proposition d'intervention ciblant les filles, ou au personnel affecté au contact direct avec les filles, en quête de nouvelles idées sur la manière de renforcer les activités de son programme. Ses outils peuvent convenir à l'inauguration d'un programme qui ciblerait pour la première fois les filles, ou à un programme féminin existant qui chercherait à se consolider ou à s'étendre. L'ouvrage s'organise en trois grandes sections : la première parle de structure, la deuxième est consacrée au contenu (y compris la santé) et la troisième se concentre sur le suivi et l'évaluation. Chaque chapitre présente une introduction au sujet abordé, des exemples de programmes existants à l'intention des filles et quelques outils pratiques et faciles à utiliser. L'ouvrage peut être téléchargé gratuitement en anglais, en espagnol ou en français depuis http://www.popcouncil.org/ publications/books/2011_AdolGirls Toolkit_fr.asp.

Donner aux jeunes femmes le pouvoir d'initier le changement.

Cet ouvrage offre un outil de formation destiné aux jeunes femmes, pour qu'elles deviennent le catalyseur du changement positif dans leur vie et dans leur communauté. Conçu par et pour les jeunes femmes, il couvre les thèmes de la santé et des droits sexuels et génésiques, de l'estime personnelle et de l'image de soi, de la violence à l'encontre des femmes, des droits humains, de la justice économique et de la paix. Les guides d'atelier inclus dans chaque module sont conçus pour aider les jeunes femmes à animer avec succès des ateliers intéressants sans devoir re- 
courir à des " animateurs experts ". Le programme est souple et adaptable aux besoins des participantes (en ce qui concerne notamment les conférenciers spéciaux ou les personnes-ressources). Les modules peuvent être présentés seuls ou sous toute combinaison adaptée aux intérêts, besoins, contraintes de temps et ressources des participantes. L'ouvrage peut être téléchargé gratuitement en anglais, en français et en espagnol depuis http://www.worldywca. org/Resources/YWCA-Publications/Empowering-Young-Women-to-Lead-Change.

\section{CONCLUSION}

Si la charge et les risques qui pèsent actuellement sur les jeunes femmes peuvent être transformés en une " double opportunité " par réforme et création d'un système de santé plus équitable et sensible aux besoins des adolescentes et des jeunes femmes vulnérables dans leur double rôle de consommatrices et prestataires de soins de santé, le sexe féminin, les communautés et les nations se développeront et s'épanouiront. L'investissement dans les filles et les jeunes femmes vulnérables en tant que consommatrices et prestataires servira les filles et les communautés par stimulation de la demande et de l'offre d'information et de services de santé de qualité adaptés au genre et à l'âge à tous les niveaux mais surtout au niveau primaire, où se trouvent souvent les plus vulnérables. La formation, la mobilisation et la rémunération des filles et des jeunes femmes au titre d'agents de santé communautaire façonneront une nouvelle ressource humaine cruciale, pour aider à relier les communautés au secteur sanitaire et améliorer les résultats de santé au profit des filles et des communautés dans leur ensemble.

\section{NOTES ET RÉFÉRENCES}

1 Organisation mondiale de la Santé. 2009. Les femmes et la santé : La réalité d'aujourd'hui, le programme de demain. Genève.
2 Temin, M. et R. Levine. 2009. Start with a Girl: A New Agenda for Global Health. Washington, DC : Center for Global Development.

3 Fonds des Nations Unies pour l'enfance. 2011. La Situation des enfants dans le monde. 2011. New York : UNICEF.

4 Ibid.

5 Levine, R. 2007. Case Studies in Global Health: Millions Saved. Boston : Jones and Bartlett.

6 Organisation mondiale de la Santé. 2003. Orientations stratégiques pour améliorer la santé et le développement des enfants et des adolescents. Genève.

7 Lloyd, COB (ed). 2005. Growing Up Global: The Changing Transitions to Adulthood in Developing Countries. Washington, DC : The National Academies Press.

8 Lloyd, C.B. 2009. New Lessons: The Power of Educating Adolescent Girls. A Girls Count Report on Adolescent Girls. New York : Population Council.

9 Fonds des Nations Unies pour l'enfance. 2008. La Situation des enfants dans le monde 2009 : La santé maternelle et néonatale. New York : UNICEF.

10 Lloyd (2005) op. cit.

11 Mensch, Barbara, Judith Bruce et Margaret E. Greene. 1998. The Uncharted Passage: Girls' Adolescence in the Developing World. New York : Population Council.

12 Nahar Q. et R. Ahmed. 2006. Addressing Special Needs of Girls' Challenges in School. Présenté à SACOSAN II. Islamabad, Pakistan.

13 Organisation mondiale de la Santé. 2011. An Update on WHO's Work on Female Genital Mutilation. Genève.

14 UNICEF (2011) op. cit.

15 Ibid

16 Agosti, J.M. et S.J Goldie. 2007. " Introducing HPV vaccine in developing countries-Key challenges and issues ", New England Journal of Medicine 356: 1908-1910.

17 Garcia-Moreno, C. et al. 2005. WHO Multicountry Study on Women's Health and Domestic Violence. Genève : Organisation mondiale de la Santé.
18 Khan M.E. et al. 2008. A Situation Analysis of Care and Support for Rape Victims at First Point of Contact in India and Bangladesh. New York : Population Council.

19 Lloyd (2005) op cit.

20 Ibid.

21 Piwoz, E. et E. Greble. 2000. HIV/AIDS and Nutrition: A Review of the Literature. Washington, DC.

22 Organisation mondiale de la Santé. 2009. Les femmes et la santé: La réalité d'aujourd'hui, le programme de demain. Genève.

23 ONUSIDA. 2008. Rapport sur l'épidémie mondiale de sida. Genève.

24 Global AIDS Alliance. 2010. Violence against Women and Children and HIV/ AIDS. Washington, DC.

25 Banque mondiale. 2006. World Development Report 2007: Development and the Next Generation. Washington, DC.

26 United Nations Platform for Action Committee. "Women \& the Economy: Globalization and Migration." http://www. unpac.ca/economy/g_migration.html.

27 OMS (2009) op. cit.

28 Ibid.

29 Organisation mondiale de la Santé. 2007. Sifting the Evidence: Gender and Tobacco Control. Genève.

30 Austrian, K. et D. Ghati. 2010. Conception d'un programme centré sur les filles. Kenya : Population Council.

31 Organisation mondiale de la Santé. 2002. Services de santé adaptés aux adolescents : Un programme pour le changement. Genève.

32 Organisation mondiale de la Santé. 2010. Guide pratique pour les soins aux adolescents : Un outil de référence destiné aux agents de la santé de premier niveau. Genève.

33 Organisation mondiale de la Santé, Commonwealth Medical Association Trust et UNICEF. 2006. Programme d'Orientation sur la Santé des Adolescents destiné aux Prestataires de Soins de santé. Genève.

34 OMS (2009) op. cit.

35 B Rabin, L., B.J. Brabin. 2005. " HIV, malaria and beyond: Reducing the disease burden of female adolescents ", Malaria Journal. 2005, 4:2. 
36 OMS (2009) op. cit.

37 Programme Abriendo Oportunidades. 2011. Guatemala : Population Council.

38 Catino, J., A. Colom et M.J. Ruiz. 2011. Equipping Mayan Girls to Improve Their Lives. New York : Population Council.

39 UNICEF. 2009. A Study on Violence against Girls: Report on the International Girl Child Conference. La Haye, Pays-Bas.

40 Programme Abriendo Oportunidades. 2010. Guatemala : Population Council.

41 Un seul programme. 2009. New York: Population Council.

42 Puntos de Encuentro: Sexto Sentido. Nicaragua. <http://www.sextosentidotv. com>.
43 Because I am a Girl: Digital and Urban Frontiers: Girls in a Changing Landscape. 2010. Royaume-Uni : Plan International.

44 "Mobile Alliance for Maternal Action". <http://www.mobilemamaalliance.org>

45 Bruce, J. 2008. The 12-Year Old CheckIn. New York : Population Council.

46 Haberland, N. "Supporting married girls: Calling attention to a neglected group ". Transitions to Adulthood. New York : Population Council. Décembre 2007.

47 Barker G., C. Ricardo et M. Nascimiento. 2007. Engaging Men and Boys in Changing Gender-Based Inequity in Health: Evidence from Program Interventions. Genève : OMS et Promundo.
48 Bruce, J. et al. 2011. First Generation of Gender and HIV Programs: Seeking Clarity and Synergy. New York : Population Council.

49 Programme Abriendo Oportunidades. 2011. Guatemala : Population Council.

50 Provide: Strengthening Youth Friendly Services. 2008. Londres : IPPF.

51 Programme Abriendo Oportunidades. 2011. Guatemala : Population Council.

52 Erulkar, Annabel. 2008. "Evaluation of Berhane Hewan Program in Addis Ababa, Ethiopia ". Population Council. 



\section{(2) Population Council}

Le Population Council s'attelle aux questions cruciales de la santé et du développement - qu'il s'agisse d'endiguer la progression du VIH, d'améliorer la santé reproductive ou d'offrir aux jeunes la promesse d'une vie satisfaisante et productive. Par ses travaux de recherche en biomédecine, en sciences sociales et en santé publique dans 50 pays, il s'efforce d'apporter avec ses partenaires des solutions qui conduisent à des politiques, des programmes et des technologies plus efficaces, pour améliorer la vie partout dans le monde. Fondé en 1952, le Population Council est une organisation non gouvernementale à but non lucratif. II siège à New York sous la conduite d'un conseil d'administration international.

www.popcouncil.org 